Canad. Math. Bull. Vol. 20 (4), 1977

\title{
PROBABILISTIC PROOFS OF THE LITTLE RIESZ THEOREM
}

\author{
BY \\ A. N. AL-HUSSAINI
}

The following theorem is a widely used corollary of the Thorn-Riesz convexity theorem ([1], pp. 526).

Theorem. Let $(\Omega, a, \mu)$ be a positive measure space, and let

$$
T: L_{1}(\Omega, a, \mu) \rightarrow L_{1}(\Omega, a, \mu)
$$

be a linear operator with $\|T\|_{1} \leq 1,\|T\|_{\infty} \leq 1$. Then $\|T\|_{p} \leq 1,1 \leq p \leq \infty$.

When $\mu$ is finite an independent proof of this theorem is given in the recent book of H. H. Schafer ([5], pp. 344).

The proof makes use of the representation of AL-spaces, and the so-called modulus of a given operator.

In this note we will provide, in detail, a proof based on a Markov process associated with $T$.

As we remark later, this approach yields through a well known technique of finitation and approximation another proof with a wider scope.

For the proof we need the following lemma.

Lemma. Let $T$ be a bounded linear operator in $L_{1}$ whose $L_{\infty}$ norm is also finite. Then there exists a positive linear operator $|T|$, the modulus of $T$, whose $L_{1}$ and $L_{\infty}$ norms do not exceed those of $T$ and such that:

$$
\begin{gathered}
|T g| \leq|T||g|, \quad g \in L_{1} \\
|T| h=\sup _{|g| \leq h}|T g|, \quad 0 \leq h \in L_{1} .
\end{gathered}
$$

Proof. See [1] and [5].

In the proof below, we utilize the Stone representation of $L_{\infty}(\Omega, \alpha, \mu)$. Plainly, this maps members of $a$ (modulo sets of measure zero) onto the

Received by the editors March 9, 1977 and revised May 13, 1977. 
algebra of closed-open subsets of a compact totally disconnected topological space (Stone space) $\Omega_{s}$. Denote this map by $S$, and write

$$
S(A)=A_{s} \text { or } S\left(1_{A}\right)=1_{A_{s}}, \quad A \in a,
$$

where $l_{A}$ denotes the indicator function of $A$. Also let $\mu_{s}\left(A_{s}\right)=\mu(A)$.

$\mu_{s}$ extends to the Baire sets $a_{s}$ of $\Omega_{s}, S$ extends to $L_{\infty}(\Omega, a, \mu)$, and each $a_{s}$ measurable function is equal (a.e. $\mu_{s}$ ) to a continuous function on $\Omega_{s}$. These statements can be justified in a variety of ways. See for example (pp. 120-121, [3]).

Finally let $|T|_{s}^{*} f_{s}$ be the continuous representative of $S\left(|T|^{*} f\right)$; then $|T|_{s}^{*} l_{A_{s}}$ is a transition function on $\Omega_{s}(p p .120,[3])$, or alternatively by (pp. 118, [4]). By construction of $\mu_{s}, S$ is $L_{p}$-norm preserving.

\section{Proof of Theorem}

CASE (1). Assume $\mu$ is a probability measure. Let $|T|$ be the modulus of $T$ as described in the lemma. From $\|T\|_{\infty} \leq 1$, it follows that $|T| 1 \leq 1$. Let $|T|^{*}$ be the adjoint of $|T|$. Then $|T|^{*}$ is a sub-Markovian operator [5], and $P(x, A)=$ $|T|^{*} l_{A}(x)$ is a sub-Markovian Kernel.

However $P(x, A)$ is not necessarily a transition Kernel. It would be the case if for example $a$ is countably generated. By passing to the Stone representation of $L_{\infty}(\Omega, a, \mu)$, we may and do assume that $P(x, A)$ is a transition Kernel and that $P(x, \Omega)=1$. If the last condition was not the case, we simply adjoin an extra point to $\Omega$. Now let $X_{0}, X_{1}, X_{2}, \ldots$ be a Markov process, corresponding to $P$ and initial distribution $\mu$. For $0 \leq f \in L_{P}$

and

$$
\left(|T|^{*} f\right)\left(X_{0}\right)=E\left\{f\left(X_{1}\right) \mid X_{0}\right\}
$$

$$
\left(|T|^{*} f\right)^{p}\left(X_{0}\right) \leq E\left\{f^{p}\left(X_{1}\right) \mid X_{0}\right\}
$$

by Jensen's inequality.

$$
\int\left(|T|^{*} f\right)^{p}(x)=E\left(|T|^{*} f\right)^{p}\left(X_{0}\right) \leq E f^{p}\left(X_{1}\right)=E\left\{E\left\{f^{p}\left(X_{1}\right)\right\} \mid X_{0}\right\} .
$$

The last quantity is equal to

$$
\int_{\Omega} f^{p}(x) \int_{Z} P(z, d x) d \mu(z),
$$

where $Z=\Omega$. But

$$
\begin{gathered}
\int_{Z} P(z, A) d \mu(z) \text { the probability of } X_{1} \in A \\
\leq \int_{Z} l_{A}(z) d \mu(z) \\
=\mu\left(X_{0} \in A\right)=\mu(A)
\end{gathered}
$$


using $|T| 1 \leq 1$, and conclude

$$
\int\left(|T|^{*} f\right)^{p}(x) d \mu(x) \leq \int f^{p}(x) d \mu(x) .
$$

For an arbitrary $f \in L_{p}$, we may use positivity of $|T|^{*}$ and obtain $(*)$ in general by replacing $f$ by $|f|$. It is easy to see that the same inequality holds if $|T|^{*}$ is replaced by $|T|$. As the Stone representation is norm preserving the result holds for the original $|T|$ and hence for $T$.

As the referee points out, since $|T|$ itself is submarkovian, we could have worked with $|T|$ instead of $|T|^{*}$, thereby avoiding the passage to $|T|$ at the end of the proof.

CASE (2). In general, i.e. when $\mu$ is a positive measure, we argue as follows: since we are dealing with integrable functions, we may and do assume that $\mu$ is $\sigma$-finite. Let $U_{1} \subset U_{2} \subset \cdots \rightarrow \Omega$ be measurable sets satisfying $\mu\left(U_{n}\right)<+\infty$, $n=1,2, \ldots$ replacing $T$ by $1_{U_{n}} T 1_{U_{n}}$ where $1_{U_{n}}$ is the indicator function of $U_{n}$, and $f$ by $1_{U_{n}} f$ we conclude that $\left\|1_{U_{n}} T 1_{U_{n}} f\right\|_{p} \leq\left\|1_{U_{n}} f\right\|_{p}$ by case (1). Thus

$$
\|T f\|_{p}=\lim _{n}\left\|1_{U_{n}} T 1_{U_{n}} f\right\|_{p} \leq\|f\|_{p}
$$

as is easily verified.

Remark. The theorem can be proved, by first proving it when $T$ is $n \times n$ matrix (special case of the case (1) of the theorem), then one may pass to the general case by approximation using conditional expectation argument. This method of finitization and approximation is not new, it takes various forms. The merit of it is the relative easiness of the finite case. The scope of such a method, including the form we used, is very wide and can be applied to handle many interpolation problems. One of these would the Thorn-Riesz Theorem itself $([1],[2])$.

\section{REFERENCES}

1. N. Dunford and Schwartz, Linear Operators, Part I., J. Wiley, 1958.

2. G. H. Hardy, J. E. Littlewood, and G. Polya, Inequalities. Cambridge University Press, 1952.

3. H. E. Lacey, The isometric theory of classical Banach spaces, Berlin, Springer-Verlag, 1974.

4. M. Rosenblatt, Markov processes; structure and asymptotic behavior, Berlin, Springer-Verlag, 1971.

5. H. H. Schaefer, Banach Lattices and Positive Operators. Springer-Verlag, 1974.

DePARTMENT OF MATH.

UNIVERSITY OF Alberta

Edmonton, Alberta T6G 2G1 